\title{
BAYESIAN SPARSE GRAPHICAL MODELS FOR CLASSIFICATION WITH APPLICATION TO PROTEIN EXPRESSION DATA
}

\section{APPENDIX A: POSITIVE DEFINITENESS CONSTRAINT}

According to our parametrization $\boldsymbol{C}=\boldsymbol{A} \odot \boldsymbol{R}$, we need to ensure the positive definiteness of $\boldsymbol{C}$ while sampling $\boldsymbol{A}$ and $\boldsymbol{R}$. The condition of positive definiteness, $I\left(\boldsymbol{C} \in \mathbb{C}_{p}\right)$, is incorporated in the priors of $\boldsymbol{A}$ and $\boldsymbol{R}$. The critical step in ensuring the positive definiteness of $\boldsymbol{C}$ is identifying the range of values of $C_{i j}$ that ensure $\boldsymbol{C}$ is positive definite given the other parameter values in our MCMC iteration. We initialize both $\boldsymbol{A}$ and $\boldsymbol{R}$ to be identity matrices.

We follow the method of Barnard McCulloch and Meng (2000), who used a similar approach, albeit in a different context. In our framework, we illustrate this method by finding the range of values of a specific element of $\boldsymbol{C}$ say $C_{12}$, to ensure that it is a positive definite matrix. Replacing $C_{12}$ by $x$ we have

$$
\boldsymbol{C}=\left(\begin{array}{cccc}
1 & x & \ldots & C_{1 p} \\
x & 1 & \ldots & C_{2 p} \\
\vdots & \vdots & \ddots & \vdots \\
C_{1 p} & C_{2 p} & \ldots & 1
\end{array}\right)
$$

It can easily be shown that the determinant of $\boldsymbol{C}$ is a quadratic function in $x$ that can be written as $f(\boldsymbol{C}) \equiv f(x)=|\boldsymbol{C}|=d x^{2}+e x+g$, where $d, e$ and $g$ depend on all the other elements $C_{i j}$ 's. Note that $f(\boldsymbol{C})>0$ is a sufficient (and necessary) condition for $\boldsymbol{C}$ to be positive definite. Let $u$ and $v$ be the roots of the equation $f(x)=d x^{2}+e x+g$. Figure 11 shows the 4 cases that arise in solving the range of values to be sampled for ensuring positive definiteness.

- if roots $\mathrm{u}$ and $\mathrm{v}$ are are real:

The range of valid values that $C_{12}$ can take for $C$ to belong to the space of positive definite correlation matrices is in between $[-1,1]$ and related to $d, u, v$.

if $d<0$ :. The exact expression for the range of $c_{12}$ is:

$$
\max (-1, u)<c_{12}<\min (1, v),
$$

if $d>0$ :. The exact expression for the range of $c_{12}$ is:

$$
-1<c_{12}<\max (-1, u), \& \min (1, v)<c_{12}<1
$$

imsart-aoas ver. 2011/12/06 file: supp_R_vb2.tex date: October 23, 2013 
- if the roots are complex:

if $d>0$ : As the matrix was positive definite before updating the element there exists atleast one real value for which the matrix is positive definite. Which means $f(C)>0$ for atleast one value in the space of real numbers. $f(C)$ is a quadratic equation which can be only positive or only negative when the roots are complex, which implies that $\mathrm{f}(\mathrm{C})$ is positive for all space of all real numbers.

if $d<0$ : This situation will not arise as there exists atleast one real value for which the matrix is positive definite.

When sampling $A_{12}$ and $R_{12}$ in our MCMC algorithm, we impose these constrains to ensure the positive definiteness of the resulting $\boldsymbol{C}$ for exploration within the space of valid graphical models.

\section{APPENDIX B: FULL CONDITIONAL DISTRIBUTIONS}

The full conditional distributions of all the model parameters and random variables used for our MCMC algorithm are shown below:

- Sampling of $Z_{o}^{u}, o=1, \ldots, n^{u}$ (= size of test/validation sample):

$$
\begin{aligned}
Z_{o} \mid \text { other } s & \propto\left\{\left|\boldsymbol{\Omega}^{(1)}\right|^{1 / 2} \exp \left(-\frac{1}{2}\left[Y_{o}-\boldsymbol{\mu}^{(1)}\right]^{T} \boldsymbol{\Omega}^{(1)}\left[Y_{o}-\boldsymbol{\mu}^{(1)}\right]\right)\right\}^{Z_{o}} \\
& \times\left\{\left|\boldsymbol{\Omega}^{(2)}\right|^{1 / 2} \exp \left(-\frac{1}{2}\left[Y_{o}-\boldsymbol{\mu}^{(2)}\right]^{T} \boldsymbol{\Omega}^{(2)}\left[Y_{o}-\boldsymbol{\mu}^{(2)}\right]\right)\right\}^{1-Z_{o}} \\
& \times h_{o}^{Z_{o}}\left(1-h_{o}\right)^{\left(1-Z_{o}\right)} \\
P\left(Z_{o}=1\right) & \propto h_{o}\left|\boldsymbol{\Omega}^{(1)}\right|^{1 / 2}\left\{\exp \left(-\frac{1}{2}\left[Y_{o}-\boldsymbol{\mu}^{(1)}\right]^{T} \boldsymbol{\Omega}^{(1)}\left[Y_{o}-\boldsymbol{\mu}^{(1)}\right]\right)\right\} \\
P\left(Z_{o}=0\right) & \propto\left(1-h_{o}\right)\left|\boldsymbol{\Omega}^{(2)}\right|^{1 / 2}\left\{\exp \left(-\frac{1}{2}\left[Y_{o}-\boldsymbol{\mu}^{(2)}\right]^{T} \boldsymbol{\Omega}^{(2)}\left[Y_{o}-\boldsymbol{\mu}^{(2)}\right]\right)\right\}
\end{aligned}
$$

After normalizing the above probabilities we draw $Z^{u}$ from a Bernoulli distribution. 
- Sampling of $\boldsymbol{\mu}^{(k)}, k=1,2$ :

$$
\begin{aligned}
& \boldsymbol{\mu}^{(1)} \mid \text { others } \propto \prod_{j: Z_{j}=1}\left\{\exp \left(-\frac{1}{2}\left[Y_{j}-\boldsymbol{\mu}^{(1)}\right]^{T} \boldsymbol{\Omega}^{(1)}\left[Y_{j}-\boldsymbol{\mu}^{(1)}\right]\right)\right\} \\
& \times\left\{\exp \left(-\frac{1}{2}\left[\boldsymbol{\mu}^{(1)}-\boldsymbol{\mu}_{0}^{(1)}\right]^{T} \boldsymbol{B}_{0}^{(1)}\left[\boldsymbol{\mu}^{(1)}-\boldsymbol{\mu}_{0}^{(1)}\right]\right)\right\} \\
& \boldsymbol{\mu}^{(1)} \mid \text { other } \sim N\left(\frac{\boldsymbol{\Omega}^{(1)} \sum_{j: Z_{j}=1} Y_{j}+\boldsymbol{B}_{0}^{(1)} \boldsymbol{\mu}_{0}^{(1)}}{|Z| \boldsymbol{\Omega}^{(1)}+\boldsymbol{B}_{0}^{(1)}},\left\{|Z| \boldsymbol{\Omega}^{(1)}+\boldsymbol{B}_{0}^{(1)}\right\}^{-1}\right) \\
& \boldsymbol{\mu}^{(2)} \mid \text { other } \sim N\left(\frac{\boldsymbol{\Omega}^{(2)} \sum_{j: Z_{j}=0} Y_{j}+\boldsymbol{B}_{0}^{(2)} \boldsymbol{\mu}_{0}^{(2)}}{(n-|Z|) \boldsymbol{\Omega}^{-1(2)}+\boldsymbol{B}_{0}^{(2)}},\left\{(n-|Z|) \boldsymbol{\Omega}^{(2)}+\boldsymbol{B}_{0}^{(2)}\right\}^{-1}\right)
\end{aligned}
$$

For the rest of the conditional distributions we assume that:

$\boldsymbol{Y}^{(1)}$ denote the set of samples $Y_{j}$ for which $Z_{j}=1$; and

$\boldsymbol{Y}^{(2)}$ denote the set of samples $Y_{j}$ for which $Z_{j}=0$

- Sampling of $R_{i j}^{(k)}, k=1,2$ :

First, we consider the complete conditional distribution of $R_{i j}^{(k)}$ and we have to consider a dimension changing step in the MCMC algorithm. One of the ways to perform it is to use reversible jump based MCMC model sampling scheme (Green, 1995; Wong et al., 2003). An alternative scheme, that of Carlin and Chib (1995), develops a product space for all possible model parameters and a model indexing variable. The space has constant dimensionality and hence a Gibbs sampler may be applied directly without concern for the varying dimension aspects of the model uncertainty problem. Other more specialized model space sampler for selection problems include the stochastic search variable selection (SSVS) methods (George and McCulloch, 1993, 1996; Kuo and Mallick, 1998; Dellportas et al., 2002). Here, we have used this SSVS method for covariance selection problem. The correlation parameter haS been drawn from the full conditional distribution as :

$$
\begin{aligned}
R_{i j}^{(k)} \mid A_{i j}^{(k)}=0, R_{-i j}^{(k)}, A_{-i j}^{(k)}, \boldsymbol{Y}^{(k)} \propto & \left.I_{\left\{u_{i j}\right.}(k), v_{i j}^{(k)}\right\}\left(A_{i j}^{(k)} R_{i j}^{(k)}\right) \\
R_{i j}^{(k)} \mid A_{i j}^{(k)}=1, R_{-i j}^{(k)}, A_{-i j}^{(k)}, \boldsymbol{Y}^{(k)} \propto & \left|\boldsymbol{\Omega}^{(k)}\right|^{n / 2} \exp \left\{\frac{-1}{2 \sigma^{2}} \operatorname{tr}\left\{\boldsymbol{\Omega}^{(k)} \boldsymbol{Y}^{(k)} \boldsymbol{Y}^{(k)^{T}}\right\}\right\} \\
& \times I_{\left\{u_{i j}^{(k)}, v_{i j}^{(k)}\right\}}\left(A_{i j}^{(k)} R_{i j}^{(k)}\right)
\end{aligned}
$$

where $R_{-i j}$ contains all other off-diagonal elements of $\boldsymbol{R}$ except the $i j$ th element and $A_{-i j}$ contains all elements of $\boldsymbol{A}$ except the $i j$ th el- 
ement. $I$ indicates the indicator function iimplying an Uniform distribution and the limits of the Uniform distribution $u_{i j}$ and $v_{i j}$ are chosen such that $\boldsymbol{C}=\boldsymbol{A} \odot \boldsymbol{R}$ is positive-definite and (conditionally) $u_{i j}$ and $v_{i j}$ are functions of $R_{-i j}$ and $A_{-i j}$. The individual parameter $R_{i j}$ are thus sampled either from their posterior conditional or from their pseudo- prior, depending upon the value of corresponding $A_{i j}$. This method had been followed by Kuo and Mallick (1998) and Dellaportas et al. (1998) in the context of variable selection. We use Gibbs sampling frame work to draw $A_{i j}$ and $R_{i j}$ jointly form their conditional posterior distribution (Kuo and Mallick, 1998; Dellaportas et. al., 1998). However, any equivalent algorithm such as reversible jump MCMC (Green, 1995) will provide similar results (Dellaportas et al., 1998). We use this conditional distribution to draw $R_{i j}^{(k)}$. We use a discrete bootstrap method to draw $R_{i j}^{(k)}$. We define a range of plausible values $\left[u_{i j}, v_{i j}\right]$ using a small grid length (say 0.01) and compute the (posterior) distribution of $R_{i j}^{(k)}$ on that range and draw a sample using the inverse CDF method.

- Joint Sampling of $A_{i j}^{(1)}$ and $\lambda_{i j}$ :

An important thing to note is that by the introduction of the latent variable $\lambda$ we are actually reparameterizing the model, making one of the $\boldsymbol{A}$ matrices fixed, i.e. given $\boldsymbol{A}^{(1)}$ and $\lambda, \boldsymbol{A}^{(2)}$ is fixed. So we need only 2 priors - one each on $\lambda$ and $\boldsymbol{A}^{(1)}$ since $\boldsymbol{A}^{(2)}$ is no longer a random variable given the former. For the same reason we also do not need to draw $q_{i j}^{(2)}$. To sample $A_{i j}^{(1)}$ and $\lambda_{i j}$, we need to evaluate the complete conditional distribution which is

$$
\begin{aligned}
{\left[A_{i j}^{(1)}, \lambda_{i j} \mid \text { other } s\right] } & \propto\left|\boldsymbol{\Omega}^{(1)}\right|^{n^{(1)} / 2} \exp \left\{\frac{-1}{2 \sigma^{(1)}} \operatorname{tr}\left\{\boldsymbol{\Omega}^{(1)} \boldsymbol{Y}^{(1)} \boldsymbol{Y}^{(1)^{T}}\right\}\right\} I_{\left\{u_{i j}^{(1)}, v_{i j}^{(1)}\right\}}\left(A_{i j}^{(1)} R_{i j}^{(1)}\right) \\
& \left|\boldsymbol{\Omega}^{(2)}\right|^{n^{(1)} / 2} \exp \left\{\frac{-1}{2 \sigma^{(2)}} \operatorname{tr}\left\{\boldsymbol{\Omega}^{(2)} \boldsymbol{Y}^{(2)} \boldsymbol{Y}^{(2)^{T}}\right\}\right\} I_{\left\{u_{i j}^{(2)}, v_{i j}^{(2)}\right\}}\left(A_{i j}^{(2)} R_{i j}^{(2)}\right) \\
& q_{i j}^{(1)} A_{i j}^{(1)}\left(1-q_{i j}^{(1)}\right)^{1-A_{i j}^{(1)}} \pi_{i j}^{\lambda_{i j}}\left(1-\pi_{i j}\right)^{\left(1-\lambda_{i j}\right)} \pi_{i j}^{e_{i j}-1}\left(1-\pi_{i j}\right)^{\left(f_{i j}-1\right)}
\end{aligned}
$$

and use it to jointly draw the binary variable $A_{i j}^{(1)}$ and $\lambda_{i j}$. Denote the above equation as $F_{A, \lambda}($.$) . Note here that there are only 4$ cases we need to draw for $A_{i j}^{(1)}$ and $\lambda_{i j}$ (i.e. $\left.[\{0,0\},\{0,1\},\{1,0\},\{1,1\}]\right)$. So we directly find the probabilities for each of the states as,

$$
\begin{aligned}
& P\left(A_{i j}^{(1)}=0, \lambda_{i j}=0 \mid \text { others }\right) \propto F_{A, \lambda}\left(A_{i j}^{(1)}=0, \lambda=0, A_{i j}^{(2)}=0\right) \\
& P\left(A_{i j}^{(1)}=0, \lambda_{i j}=1 \mid \text { others }\right) \propto F_{A, \lambda}\left(A_{i j}^{(1)}=0, \lambda=1, A_{i j}^{(2)}=1\right) \\
& P\left(A_{i j}^{(1)}=1, \lambda_{i j}=0 \mid \text { others }\right) \propto F_{A, \lambda}\left(A_{i j}^{(1)}=1, \lambda=0, A_{i j}^{(2)}=1\right)
\end{aligned}
$$


$P\left(A_{i j}^{(1)}=1, \lambda_{i j}=1 \mid\right.$ other $\left.s\right) \propto F_{A, \lambda}\left(A_{i j}^{(1)}=1, \lambda=1, A_{i j}^{(2)}=0\right)$.

Consequently, we sample one of the configurations $A_{i j}^{(1)}, \lambda_{i j}$ as a discrete binary variable using these probabilities as weights.

- Complete conditional distribution for $Q^{(1)}, \pi_{i j}$ and $h_{o}$ :

$$
\begin{gathered}
q_{i j}^{(1)} \mid A_{i j}^{(1)} \propto q_{i j}^{(1)^{A_{i j}^{(1)}}}\left(1-q_{i j}^{(1)}\right)^{\left(1-A_{i j}^{(1)}\right)} q_{i j}^{(1)^{\alpha_{i j}-1}}\left(1-q_{i j}^{(1)}\right)^{\left(\beta_{i j}-1\right)} \\
q_{i j}^{(1)} \mid A_{i j}^{(1)} \sim \operatorname{Beta}\left(A_{i j}^{(1)}+\alpha_{i j}, \beta_{i j}+1-A_{i j}^{(1)}\right) .
\end{gathered}
$$

Similarly

$$
\pi_{i j} \mid \lambda_{i j} \sim \operatorname{Beta}\left(\lambda_{i j}+e_{i j}, f_{i j}+1-\lambda_{i j}\right)
$$

Similarly

$$
h_{o} \mid Z_{o}^{u} \sim \operatorname{Beta}\left(Z_{o}^{u}+\eta_{o}, \zeta_{o}+1-Z_{o}^{u}\right) .
$$

- Complete conditional distribution for $S^{(k)}$

$$
\begin{aligned}
S_{i}^{(k)} \mid S_{-i}^{(k)}, \boldsymbol{Y}^{(k)}, \sigma^{2(k)} \propto & \left|S^{(k)}\left(A^{(k)} \odot R^{(k)}\right) S^{(k)}\right|^{n^{(k)} / 2} \\
& \times \exp \left\{\frac{-1}{2 \sigma^{2(k)}} \operatorname{tr}\left\{S^{(k)}\left(A^{(k)} \odot R^{(k)}\right) S^{(k)} \boldsymbol{Y}^{(k)} \boldsymbol{Y}^{(k)^{T}}\right\}\right\} \\
& \times S_{i}^{(k)^{-g-1}} \exp \left(\frac{-h}{S_{i}^{(k)}}\right) \\
\propto & S_{i}^{(k)^{n}} \exp \left\{\frac{-1}{2 \sigma^{2}(k)} \operatorname{tr}\left\{S^{(k)}\left(A^{(k)} \odot R^{(k)}\right) S^{(k)} \boldsymbol{Y}^{(k)} \boldsymbol{Y}^{(k)^{T}}\right\}\right\} \\
& \times S_{i}^{(k)^{-g-1}} \exp \left(\frac{-h}{S_{i}^{(k)}}\right) .
\end{aligned}
$$

\section{APPENDIX C: SIMULATIONS}

We performed simulations to evaluate the operating characteristics of our methodology. To make our simulations realistic we tried to mimic the real data scenario as much as as possible. We fixed the number of proteins to 50 (as in the real data) and generated data from multivariate normal distributions with the correlations between the proteins were modeled using the covariance matrix. We generated data under the following three scenarios for two groups/classes:

- scenario 1: The classes have same means and different covariance matrices. 
- scenario 2: The classes have same means and mutually exclusive covariance matrices.

- scenario 3: The classes have different means and mutually exclusive covariance matrices.

For scenario 1, the covariance matrices for the two classes were set to the estimated covariance matrices in the real data analysis and is shown in Fig 1. The means of the two classes were assigned as zero. In scenario 2 the means were kept the same but the covariance matrices were assigned to be mutually exclusive block diagonals - for the first class 30 of the 50 proteins were correlated as 3 blocks with 10 proteins in each block and the remaining 20 were independent (as shown in Fig 2). In the second class the 20 that were independent previously were correlated as 2 blocks with10 proteins in each block the remaining 30 that were correlated previously were independent in this class. For the third scenario the means for the two classes were computed from the real data of breast and ovarian cell lines which were different and the covariance structure was similar to scenario 2 . We generated 100 training data sets of 30 samples and 25 samples for class 1 and 2 respectively, under the 3 scenarios. Analogously, 100 test data sets of same dimensions were simulated for the 3 scenarios. The classification performance of the seven methods used on real data, is reported using misclassification percentages in the test datasets and is shown in Figures 3-5 for the 3 scenarios respectively. The methods compared here are SVM (Network Based support vector machines) LDA (linear discriminant analysis), KNN (K-nearest neighbor), DQDA (diagonal quadratic discriminant analysis), DLDA (diagonal linear discriminant analysis) NBC(Naive Bayes Classifier) and BGBC (Bayesian graph-based classifier), which is the method proposed in this paper. Also all the other methods dont estimate the network structure where as the BGBC estimates the network structure as well as classifies the samples. Performing a two step procedure by separately estimating the networks and then using them to classify will lead to a bias in the classification.

Overall, the results for the 3 scenarios show that accounting for network structure is essential for classification performance when the means of the two classes are very close to each other. In scenario 1, we see that our BGBC method and KNN performs the best (least median misclassification error) with all other methods achieving slightly higher misclassification rate. In scenario 2, our BGBC method outperforms all other methods, since the network structure (covariance matrix) contains most of the information regarding the separability of the classes - which is the objective of our analysis. In the third scenario, all the methods perform equivalently since the separability is dictated both by the mean as well as the covariance and even in this 

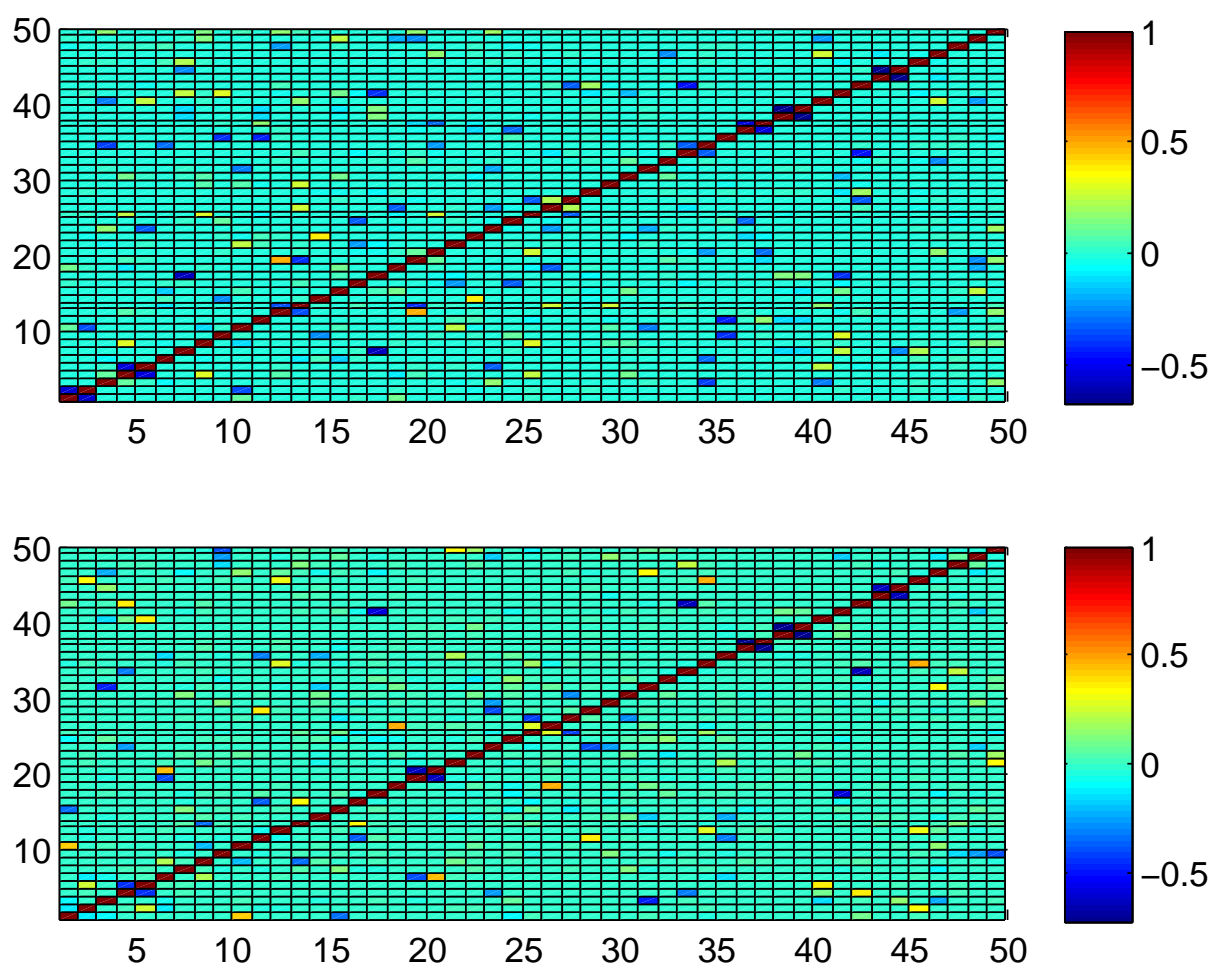

FIG 1. This figure shows the simulated precision matrices for the two classes in Scenario1

scenario there is information in the network structure which we can exploit for improving classification. 

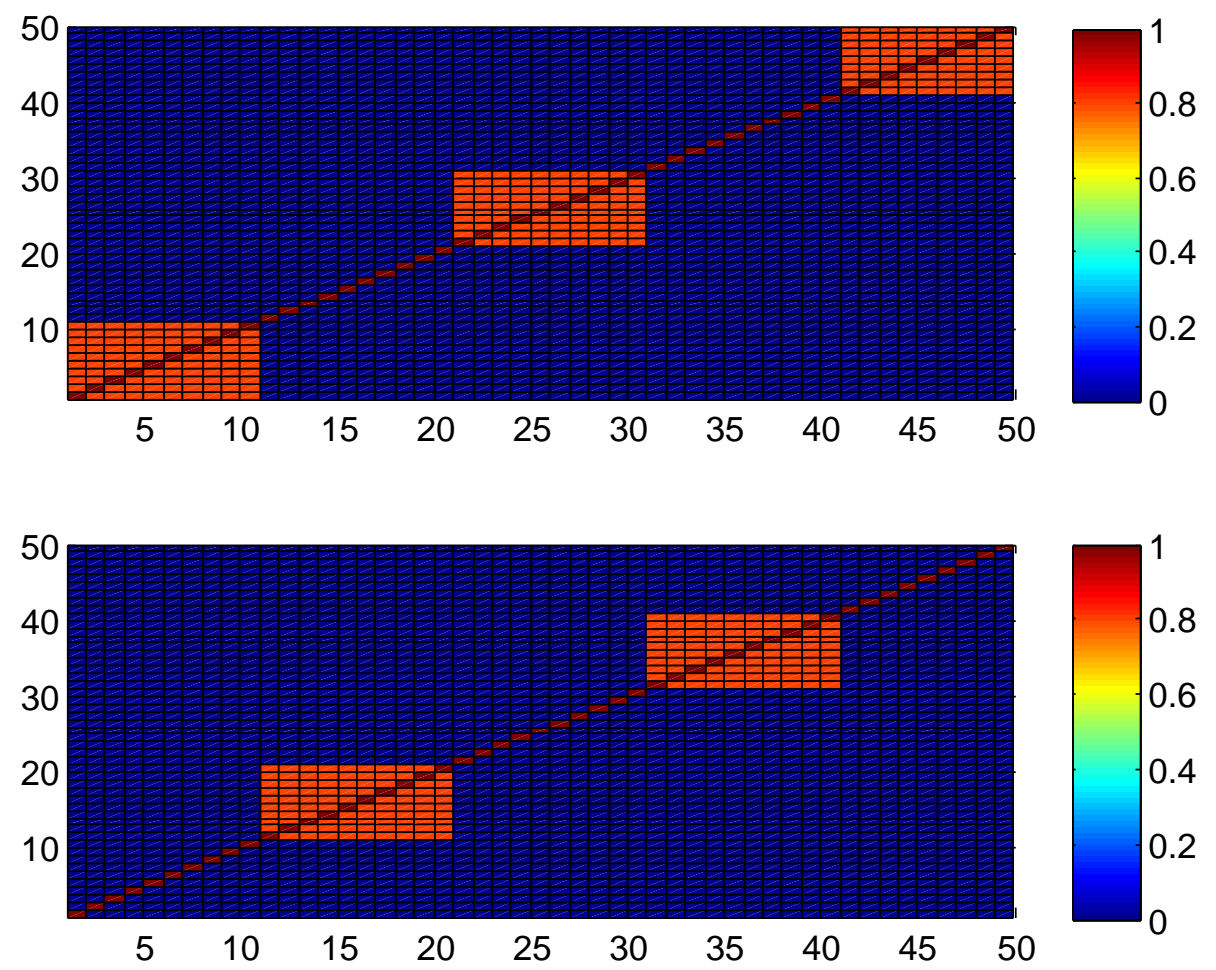

FIG 2. This figure shows the simulated precision matrices for the two classes in Scenario2,3 


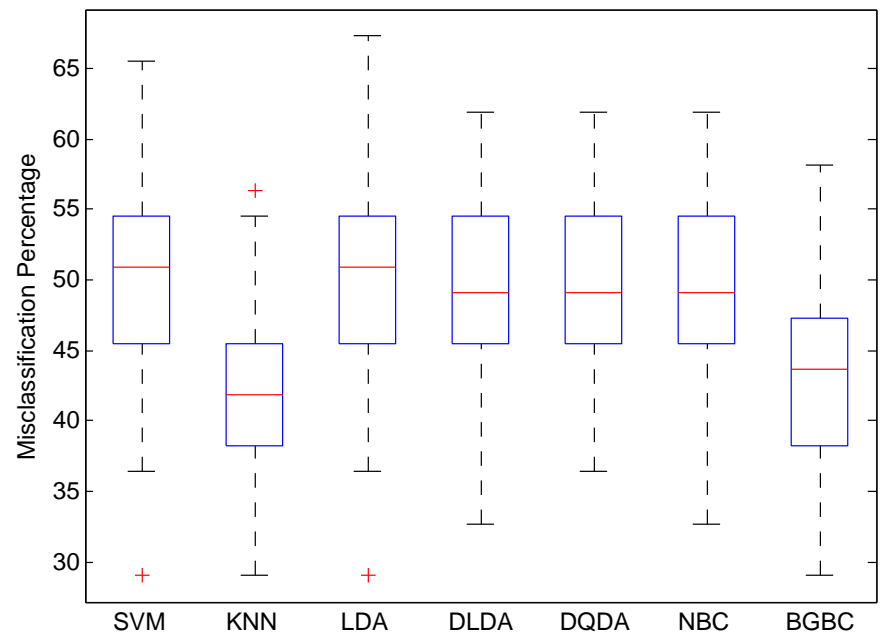

FIG 3. Misclassification error rates for different classifiers for simulated data in scenario 1. The methods compared here are SVM(Network Based Support Vector Machine), LDA (linear discriminant analysis), KNN (K-nearest neighbor), DQDA (diagonal quadratic discriminant analysis), DLDA (diagonal linear discriminant analysis) $N B C$ (Naive Bayes Classifier) and BGBC (Bayesian graph-based classifier), which is the method proposed in this paper. The mean and the standard deviation are values of the misclassification percentage over 100 random splits of the data. 


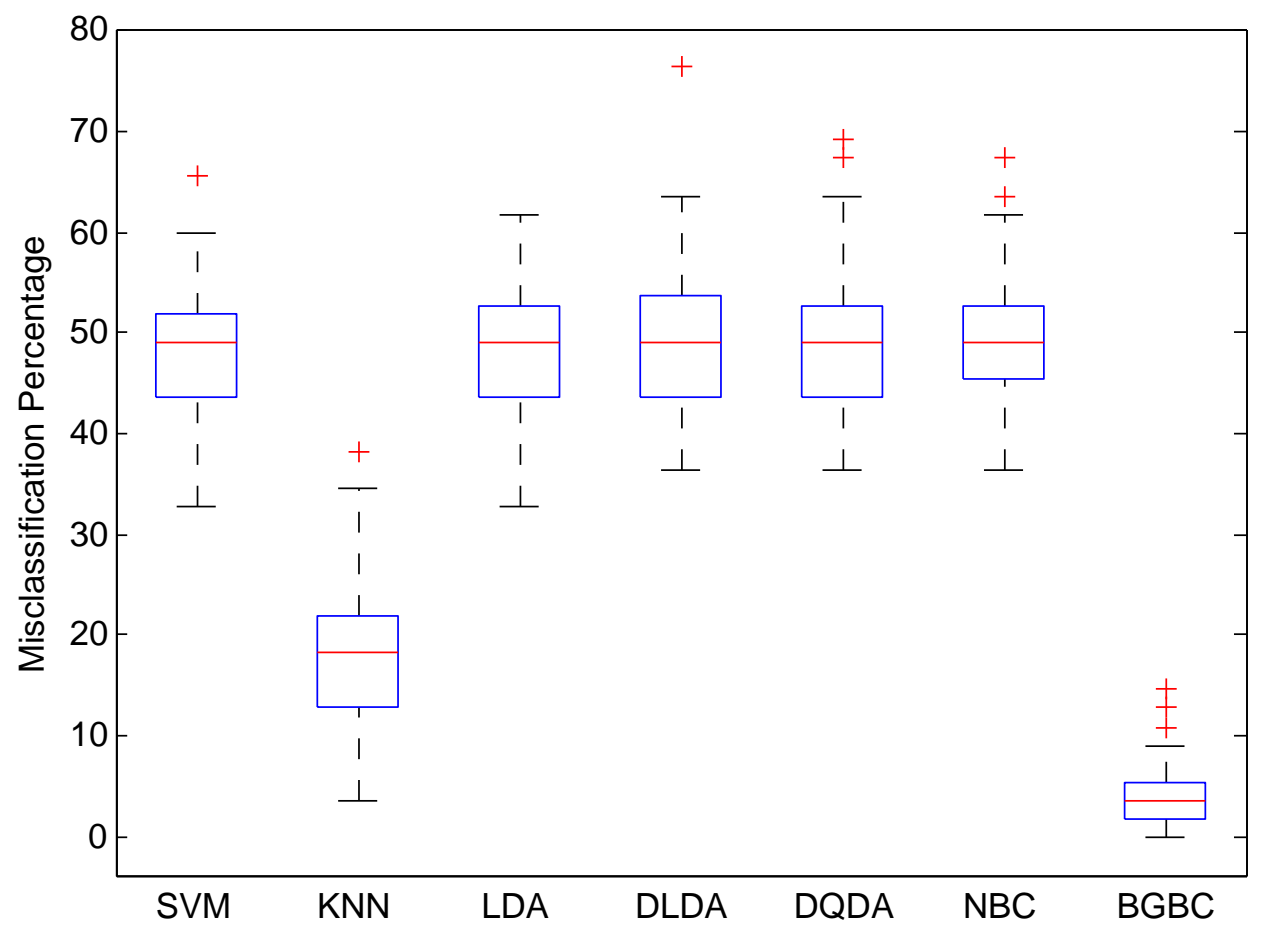

FIG 4. Misclassification error rates for different classifiers for simulated data in scenario 2. The methods compared here are SVM(Network Based Support Vector Machine), LDA (linear discriminant analysis), KNN (K-nearest neighbor), DQDA (diagonal quadratic discriminant analysis), DLDA (diagonal linear discriminant analysis) NBC(Naive Bayes Classifier) and BGBC (Bayesian graph-based classifier), which is the method proposed in this paper. The mean and the standard deviation are values of the misclassification percentage over 100 random splits of the data.

imsart-aoas ver. 2011/12/06 file: supp_R_vb2.tex date: October 23, 2013 


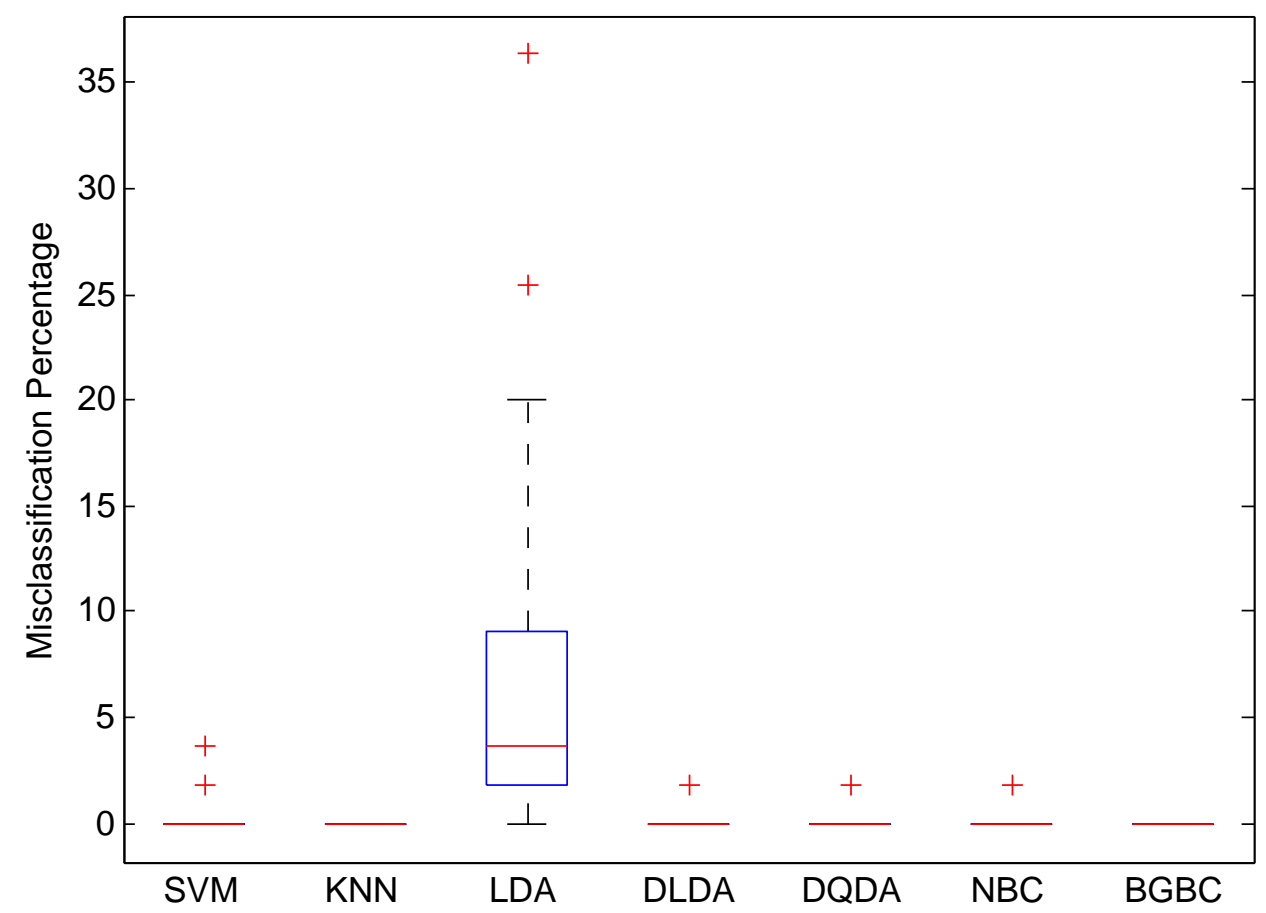

FIG 5. Misclassification error rates for different classifiers for simulated data in scenario 3. The methods compared here are SVM(Network Based Support Vector Machine), LDA (linear discriminant analysis), KNN (K-nearest neighbor), DQDA (diagonal quadratic discriminant analysis), DLDA (diagonal linear discriminant analysis) NBC(Naive Bayes Classifier) and BGBC (Bayesian graph-based classifier), which is the method proposed in this paper. The mean and the standard deviation are values of the misclassification percentage over 100 random splits of the data. 


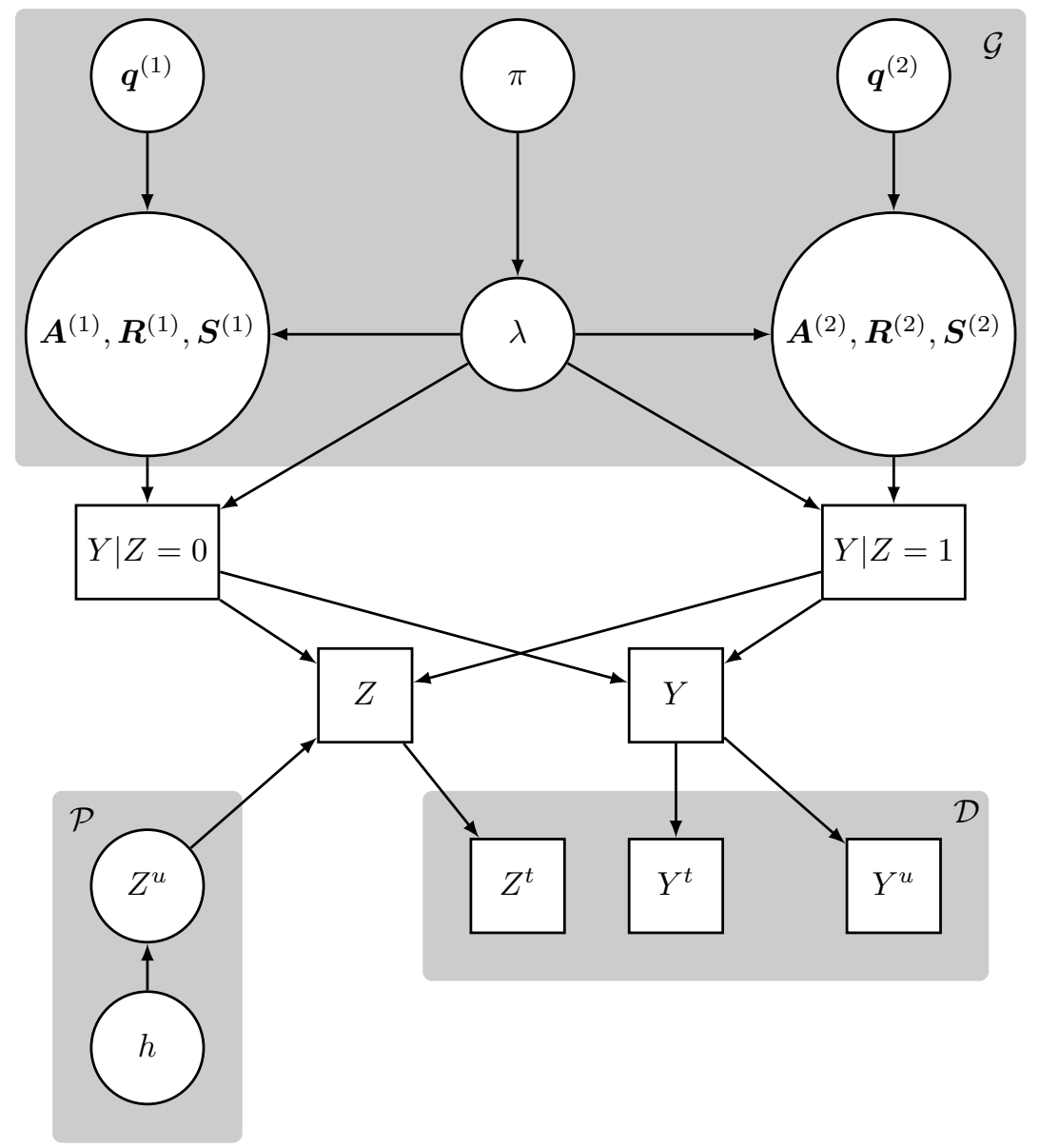

FIG 6. This figure represents the directed acyclic graph (DAG) representation of the binary graph-based classification model. The random variables are indicated by rectangles and the parameters are indicated by circles. The shaded panel indexed by $\mathcal{D}$ (bottom right) represents the observed data that are split into training and test sets denoted by $(t, u)$ subscripts respectively. The top panel indexed by $\mathcal{G}$ represents the parameters (and its dependencies) of the sparse Gaussian graphical model and the panel indexed by $\mathcal{P}$ (bottom left) represents the parameters of the prediction scheme. 


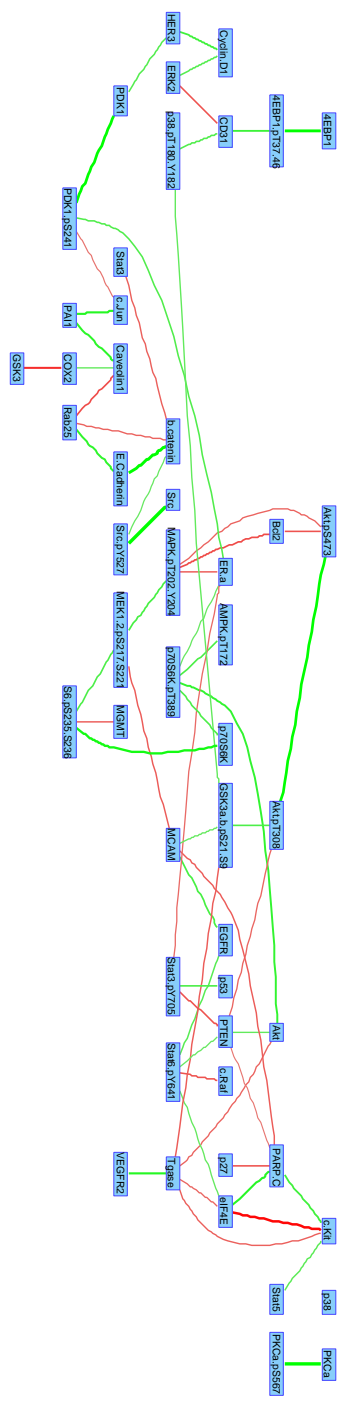

(a) Breast network with prior information

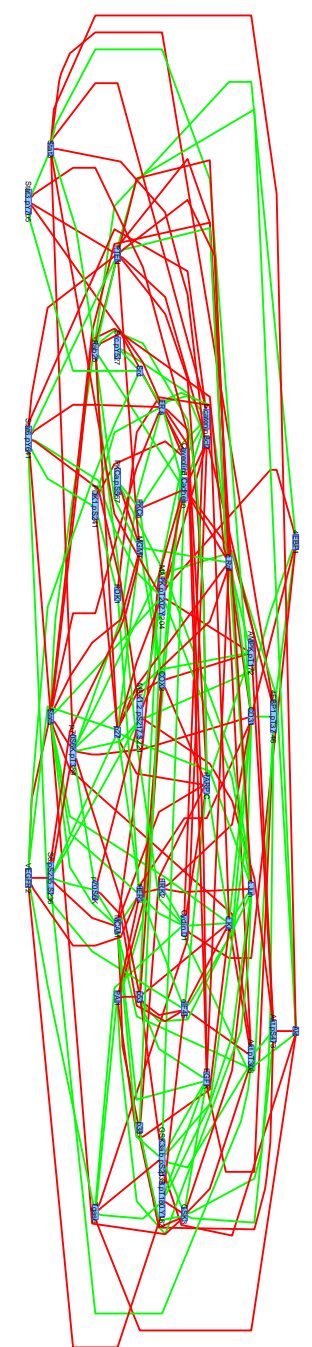

(b) Breast network without prior information

FIG 7. Significant edges for the proteins in the PI3K-AKT kinase pathway for breast cancer cell lines estimated using prior information from the pathway (left panel) and estimated without using prior information (right panel). The significant edges were computed using a Bayesian FDR of 0.10 . The red (green) lines between the proteins indicate a negative (positive) correlation between the proteins. The thickness of the edges corresponds to the strength of the associations, with stronger associations having greater thickness.

imsart-aoas ver. 2011/12/06 file: supp_R_vb2.tex date: October 23, 2013 

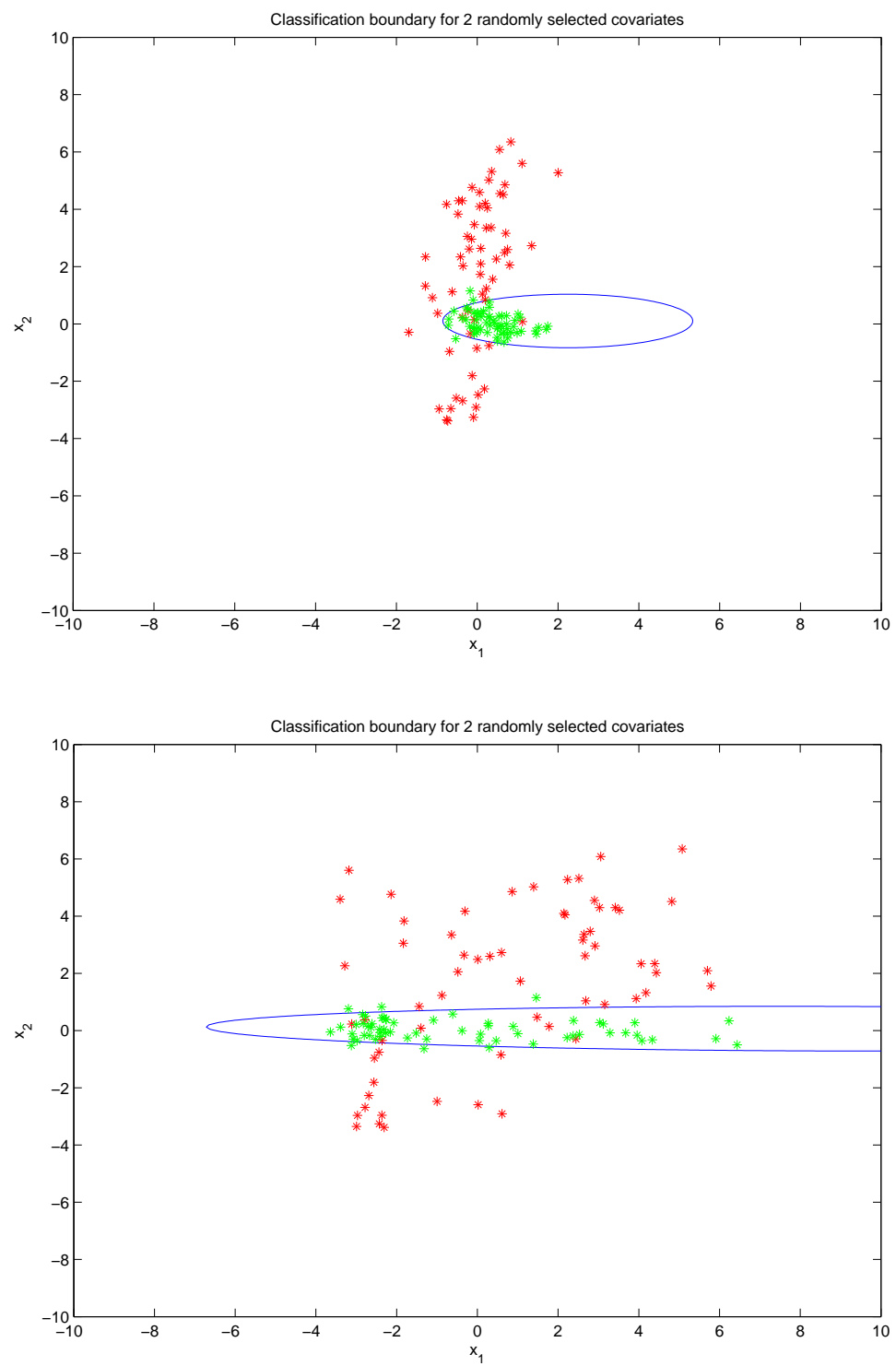

FIG 8. Nonlinear classification boundaries for two randomly selected covariates. Green points represent breast cancer cell line data and red points represent ovarian cancer cell line data. The blue line is the nonlinear classification boundary obtained using our proposed Bayesian graph based classifier.

imsart-aoas ver. 2011/12/06 file: supp_R_vb2.tex date: October 23, 2013 

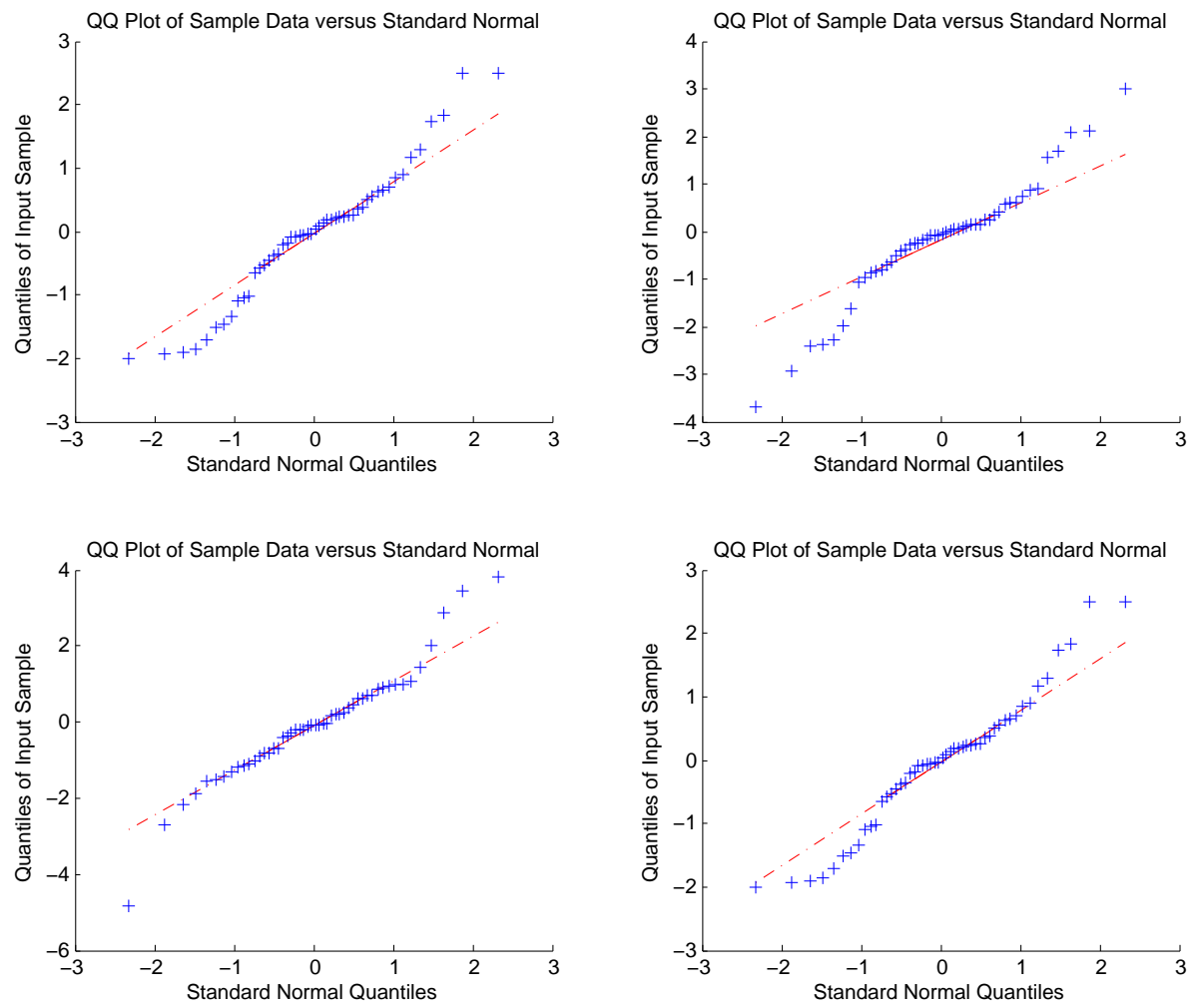

FIG 9. This figure shows the qq plots for some of the protein expressions in RPPA data. Each plots is a quantile plot of data of individuals for a protein in 50 Breast cell lines. While there are some outliers the distributions of the data justify the assumption of multivariate normality. 


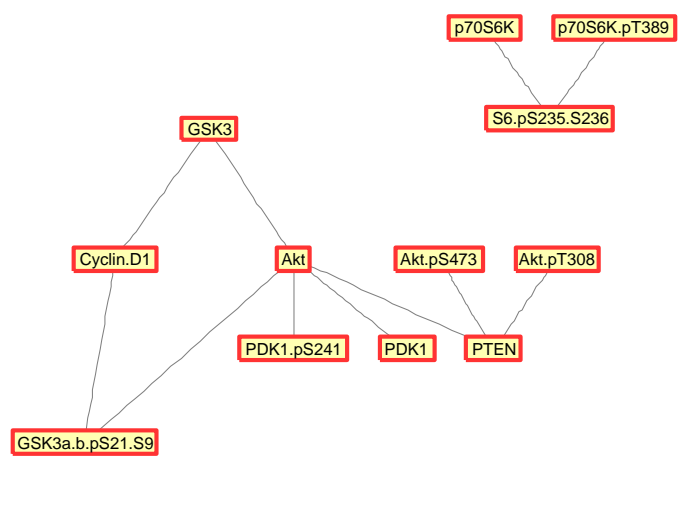

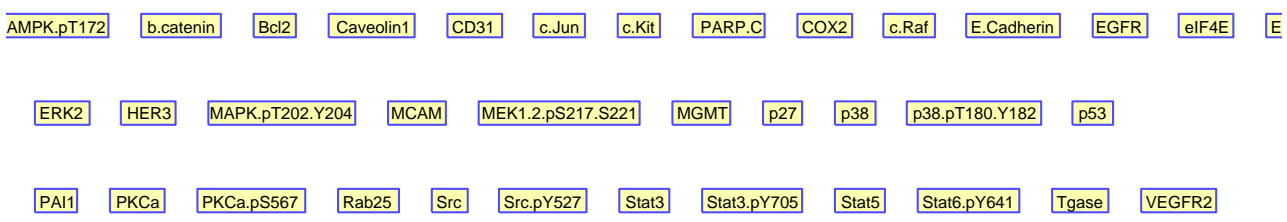

FIG 10. This figure details the prior network information derived PI3K-AKT pathway that was incorporated into our analysis. The proteins enclosed in the red boxes had inter connections with other proteins. The gray lines indicate the relationship between the proteins. All the other proteins in blue boxes were unrelated apriori.

imsart-aoas ver. 2011/12/06 file: supp_R_vb2.tex date: October 23, 2013 

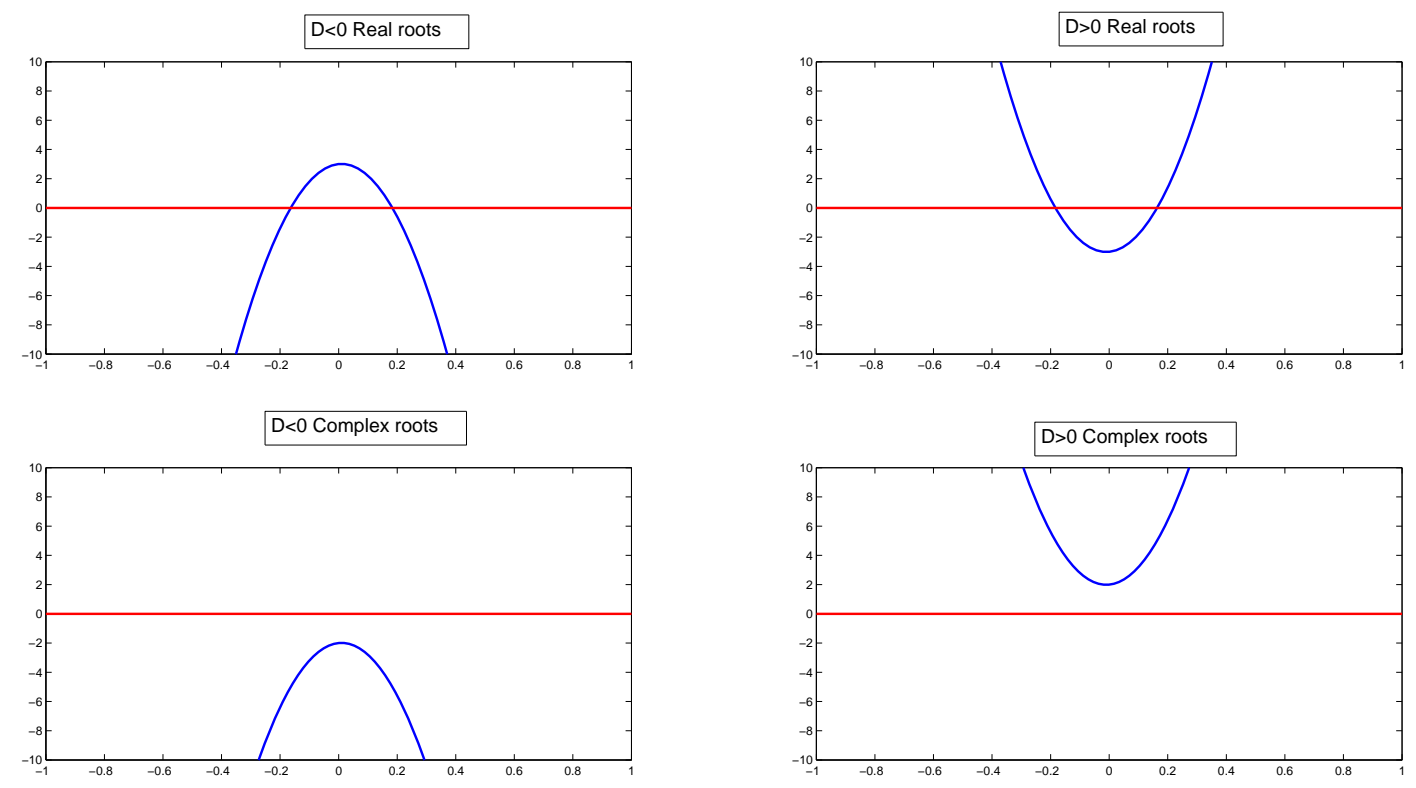

FIG 11. This figure shows the 4 cases for the roots of the quadratic equation $d x^{2}+e x+$ $f$. The range of values which preserve positive definiteness for each of the elements are described in Appendix A.

imsart-aoas ver. 2011/12/06 file: supp_R_vb2.tex date: October 23, 2013 
18<smiles>CCCCCCCC</smiles>
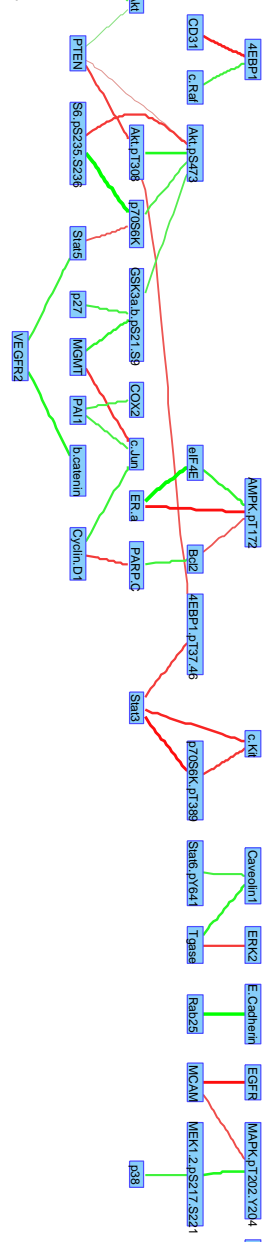

層
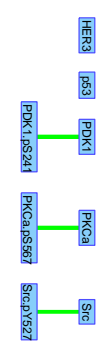

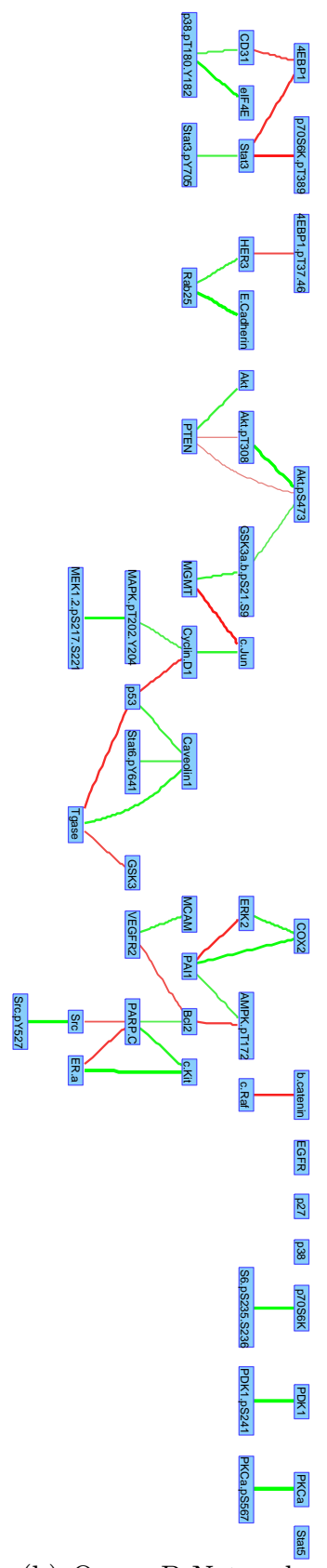

(b) Ovary-B Network

(a) Ovary-A Network

FIG 12. Significant edges for the proteins in the PI3K-AKT kinase pathway for ovarian cancer cell lines grown in three different tissue culture conditions: A, B and C (see main text) computed using a Bayesian FDR set to 0.10. The red (green) lines between the proteins indicate a negative (positive) correlation between the proteins. The thickness of the edges corresponds to the strength of the associations, with stronger associations having greater thickness.

imsart-aoas ver. 2011/12/06 file: supp_R_vb2.tex date: October 23, 2013 

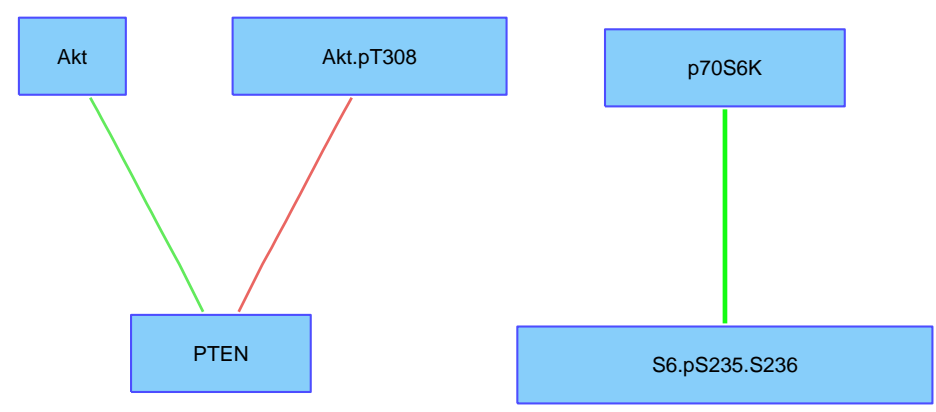

(a) Conserved network between prior and breast cancer cell lines.

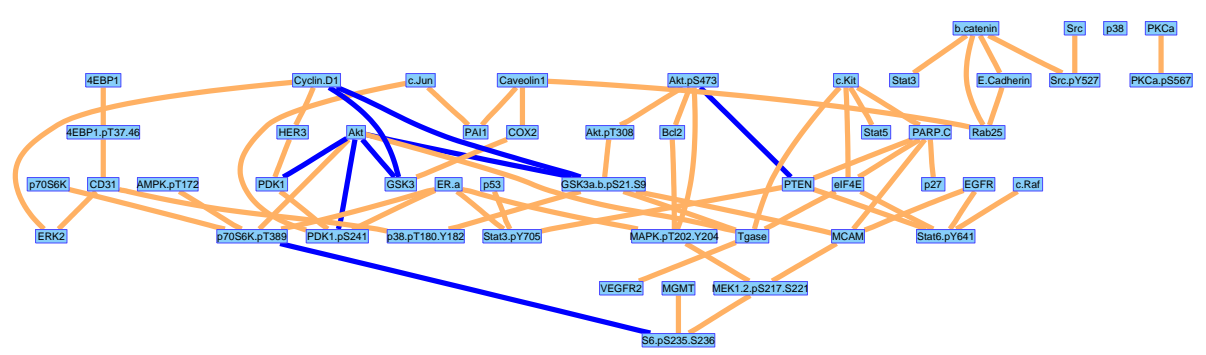

(b) Differential network between prior and breast cancer cell lines.

FIG 13. Conserved and differential networks for the proteins in the PI3K-AKT kinase pathway between prior and breast cancer cell lines. In the conserved network (top panel), the red (green) lines between the proteins indicate a negative (positive) correlation between the proteins. In the differential network (bottom row), the blue lines between the proteins indicate a relationship that was present in the prior but not in the breast cancer cell lines; the orange lines between the proteins indicate a relationship in the breast cancer cell lines but not in the prior. 

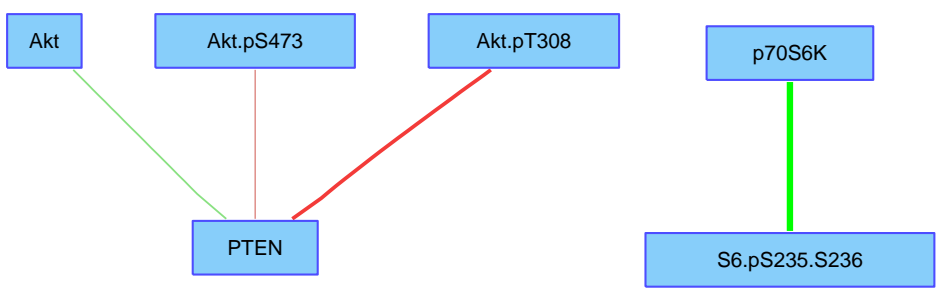

(a) Conserved network between prior and Ovary A cancer cell lines.

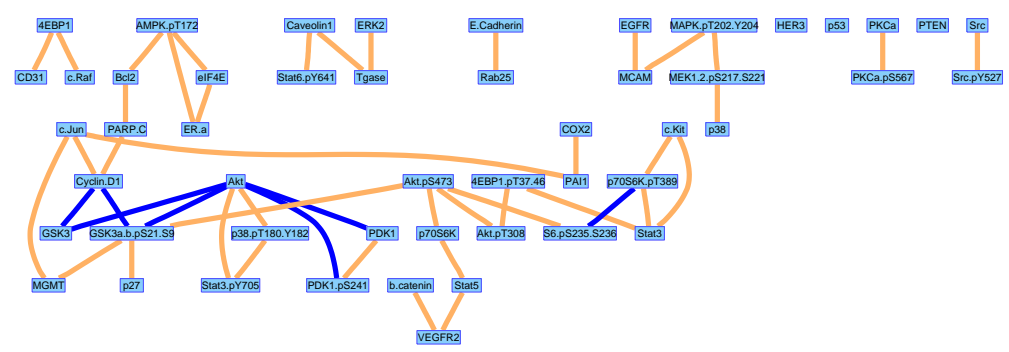

(b) Differential network between prior and Ovary A cancer cell lines.

FIG 14. Conserved and differential networks for the proteins in the PI3K-AKT kinase pathway between prior and Ovary A cancer cell lines. In the conserved network (top panel), the red (green) lines between the proteins indicate a negative (positive) correlation between the proteins. In the differential network (bottom row), the blue lines between the proteins indicate a relationship that was present in the prior but not in the Ovary A cancer cell lines; the orange lines between the proteins indicate a relationship in the OvaryA cancer cell lines but not in the prior. 


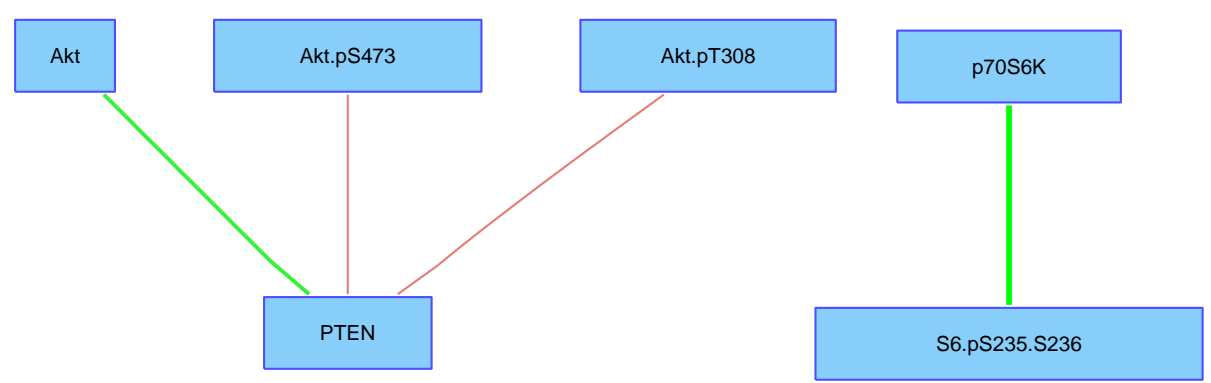

(a) Conserved network between prior and Ovary B cancer cell lines.
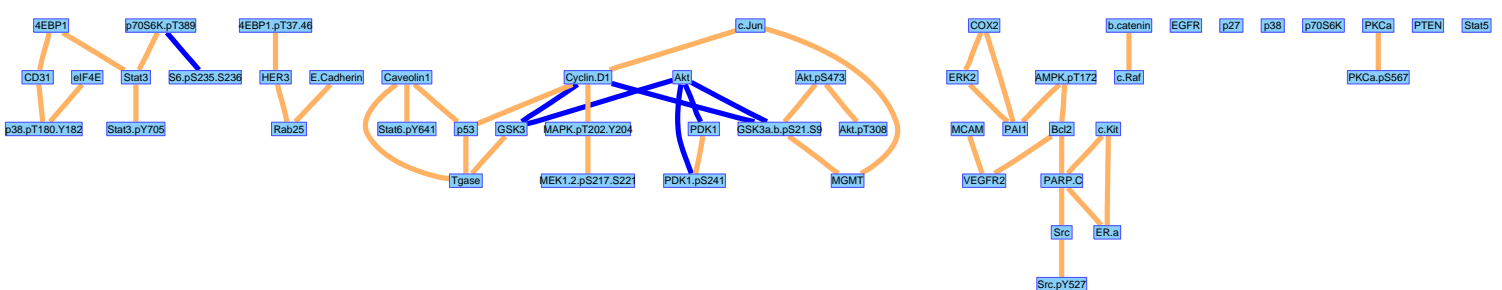

(b) Differential network between prior and Ovary B cancer cell lines.

FIG 15. Conserved and differential networks for the proteins in the PI3K-AKT kinase pathway between prior and Ovary B cancer cell lines. In the conserved network (top panel), the red (green) lines between the proteins indicate a negative (positive) correlation between the proteins. In the differential network (bottom row), the blue lines between the proteins indicate a relationship that was present in the prior but not in the Ovary $B$ cancer cell lines; the orange lines between the proteins indicate a relationship in the Ovary $B$ cancer cell lines but not in the prior. 


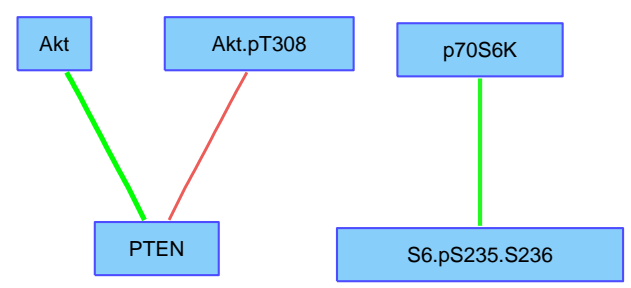

(a) Conserved network between prior and Ovary C cancer cell lines.

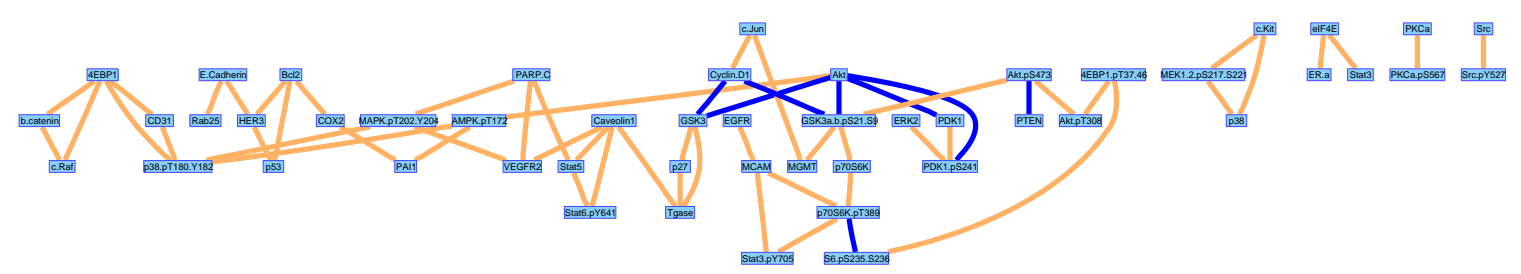

(b) Differential network between prior and Ovary C cancer cell lines.

FIG 16. Conserved and differential networks for the proteins in the PI3K-AKT kinase pathway between prior and Ovary $C$ cancer cell lines. In the conserved network (top panel), the red (green) lines between the proteins indicate a negative (positive) correlation between the proteins. In the differential network (bottom row), the blue lines between the proteins indicate a relationship that was present in the prior but not in the Ovary $C$ cancer cell lines; the orange lines between the proteins indicate a relationship in the Ovary $C$ cancer cell lines but not in the prior. 


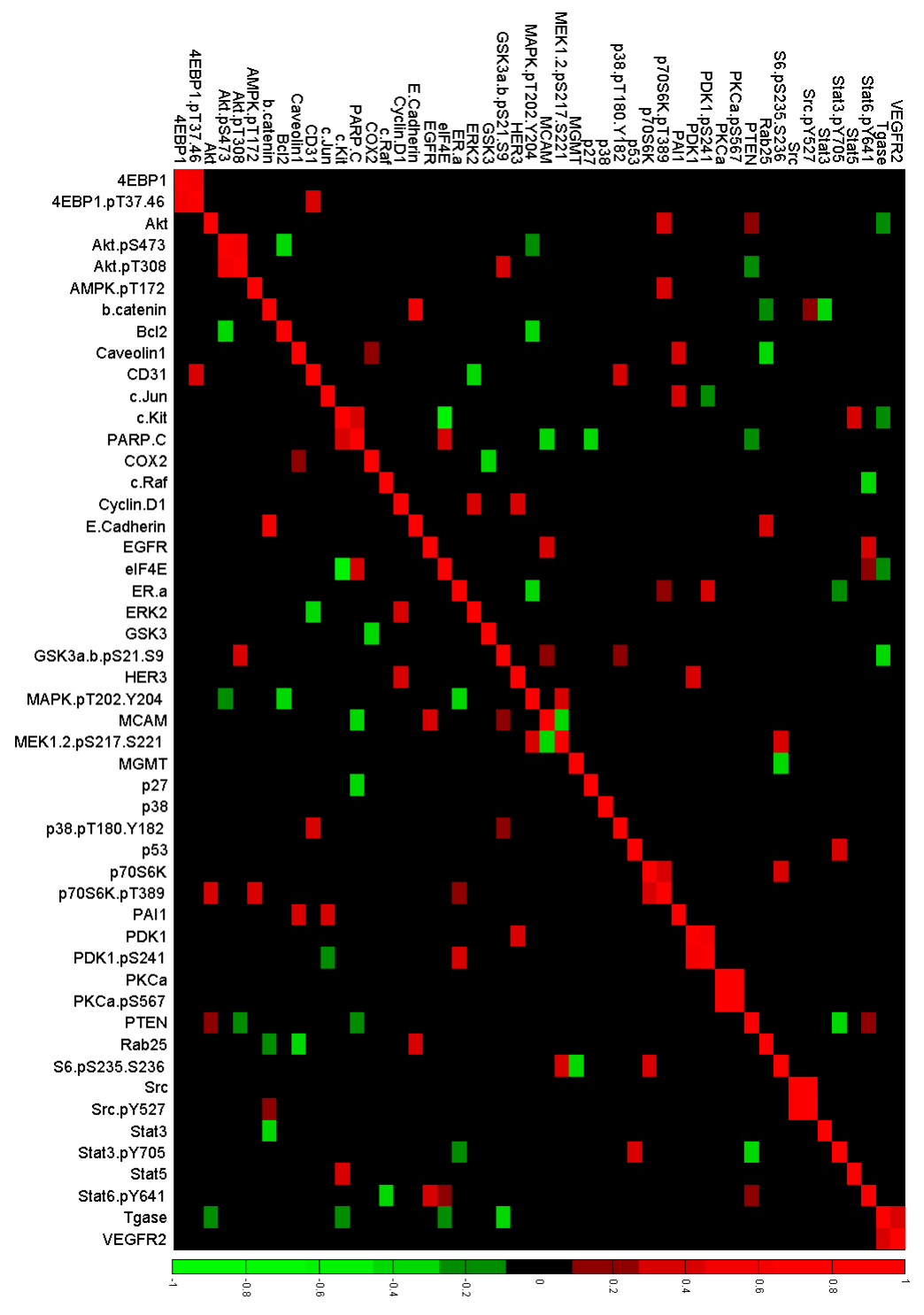

FIG 17. This figure depicts the correlation matrix (C) for Breast cancer cell lines using a heat map plot. 


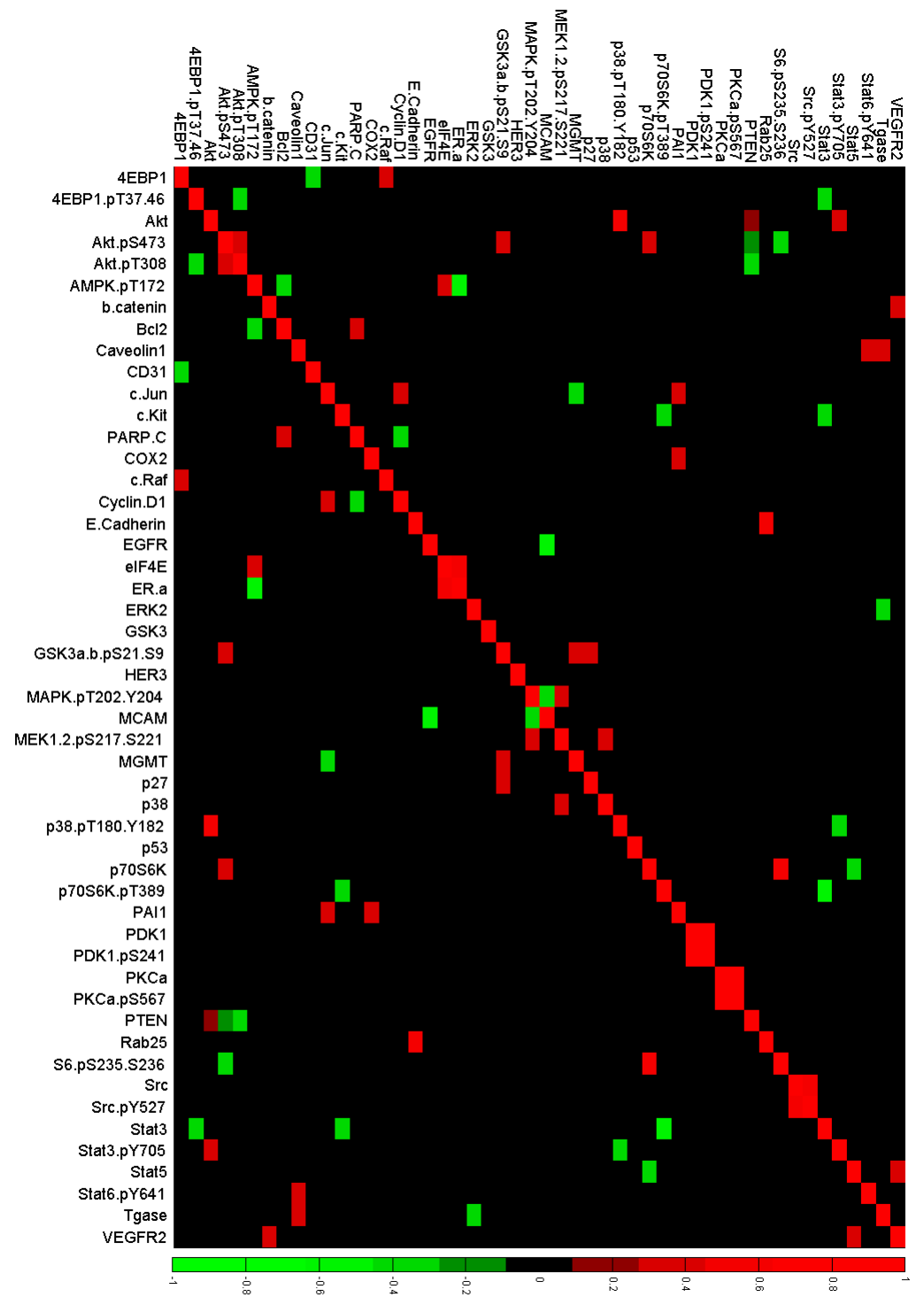

FIG 18. This figure depicts the correlation matrix (C) for Ovary A cancer cell lines using a heat map plot. 


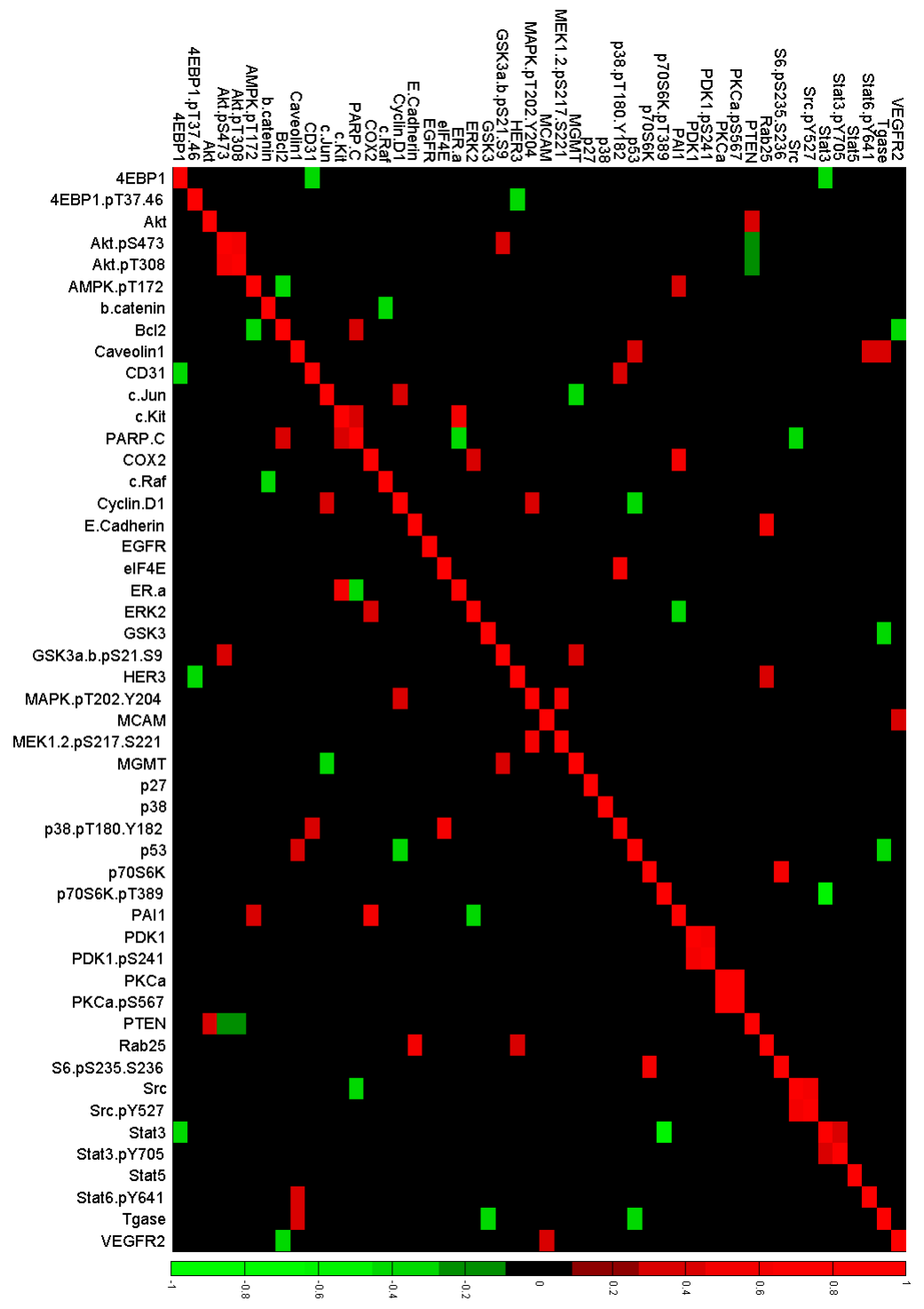

FIG 19. This figure depicts the correlation matrix (C) for Ovary B cancer cell lines using a heat map plot. 


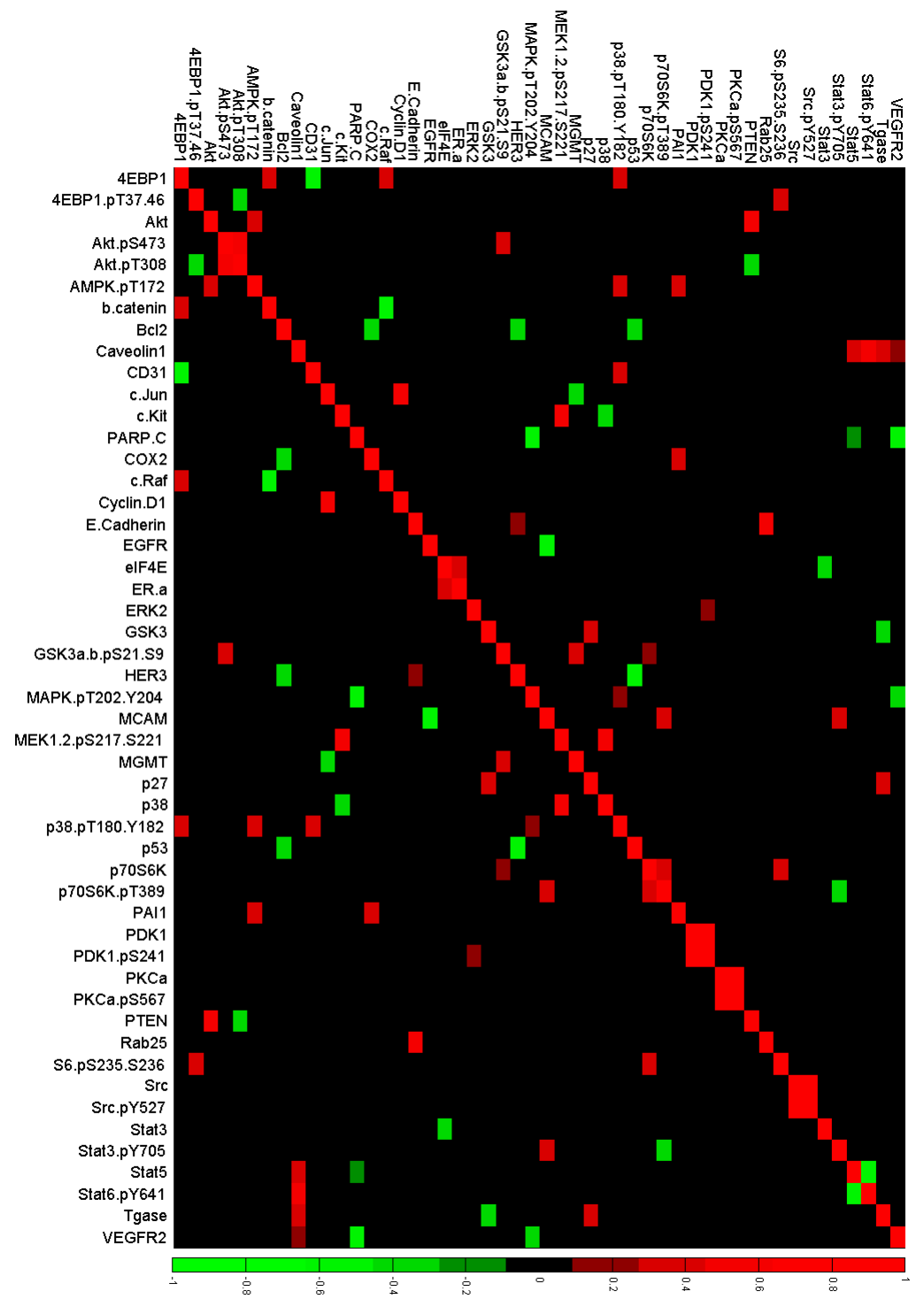

FIG 20. This figure depicts the correlation matrix $(C)$ for Ovary $C$ cancer cell lines using a heat map plot. 